\title{
Antimicrobial Dressing versus Standard Dressing in Obese Women Undergoing Cesarean Delivery: A Randomized Controlled Trial
}

\author{
Antonio F. Saad, MD ${ }^{1}$ Ashley E. Salazar, MSN, WHNP ${ }^{1} \quad$ Lindsey Allen, RN ${ }^{1} \quad$ George R. Saade, MD ${ }^{1}$ \\ ${ }^{1}$ Division of Maternal-Fetal Medicine, Department of Obstetrics and \\ Address for correspondence Antonio F. Saad, MD, Division of Critical \\ Gynecology, The University of Texas Medical Branch, Galveston, \\ Care Medicine and Maternal-Fetal Medicine, Department of Obstetrics \\ and Gynecology, University of Texas Medical Branch, 301 University \\ Boulevard, Galveston, TX 77555-0587 (e-mail: afsaad@utmb.edu).
}

Am J Perinatol 2022;39:951-958

\begin{abstract}
Keywords

- wound dressing

- cesarean

- obese

- satisfaction

- wound care

- wound complications

Objective This study aimed to evaluate the effect of a novel antimicrobial dressing on patient satisfaction and health-related quality of life (HRQoL) following a cesarean delivery. Study Design This was an open-label, single-center, two-arm randomized controlled trial. This study was done at the tertiary center, maternal unit, Galveston, TX. Pregnant women with body mass indices $\geq 35 \mathrm{~kg} / \mathrm{m} 2$ were screened for eligibility. Women were randomized to ReliaTect Post-Op Dressing (RELIATECT) or standard wound dressing (STANDARD). Primary outcome was patient satisfaction and HRQoL using validated questionnaires. Secondary outcomes were provider satisfaction, surgical site infection (SSI) rates, and wound complications.

Results In total, 160 women were randomized. Population characteristics were not significant among groups. RELIATECT dressing group had an overall higher score of satisfaction and HRQoL compared with STANDARD group. Women in the RELIATECT group reported less incision odor and incisional pain. Compared with the STANDARD group, most women in RELIATECT dressing group reported better daily activities, selfesteem, personal hygiene, body image, and sleep. Providers reported that the RELIATECT dressing allowed better assessment of the surgical incision site, allowed patients to shower early, and did observe less wound dressing leakage. No differences were found in other secondary end points.

Conclusion Postcesarean RELIATECT dressing for wound care in pregnant women with obesity had better patient and provider satisfaction as well as better HRQoL scores. Further, level 1 evidence is needed to assess its impact on SSI rates and wound complication, as this trial was not powered to accomplish this goal.
\end{abstract}

\section{Key Points}

- This study was conducted to evaluate RELIATECT on patient satisfaction and HRQoL following a cesarean.

- Post-cesarean RELIATECT dressing for wound care had better HRQoL and patient and provider satisfaction scores.

- This is the first randomized controlled trial evaluating RELIATECT dressing in obese pregnant women undergoing cesarean section.

received

September 9, 2020 accepted after revision

October 6, 2020

published online

December 2, 2020
DOI https://doi.org/ 10.1055/s-0040-1721112. ISSN 0735-1631.
(C) 2020. The Author(s)

This is an open access article published by Thieme under the terms of the Creative Commons Attribution-NonDerivative-NonCommercial-License, permitting copying and reproduction so long as the original work is given appropriate credit. Contents may not be used for commercial purposes, or adapted, remixed, transformed or built upon. (https://creativecommons.org/ licenses/by-nc-nd/4.0/)

Thieme Medical Publishers, Inc., 333 Seventh Avenue, 18th Floor, New York, NY 10001, USA 
Cesarean delivery is the most common surgical procedure in the United States. ${ }^{1}$ Despite a concerted effort to decrease the cesarean delivery rate, it continues to hover around $30 \%$ and is unlikely to decrease. ${ }^{2}$ Surgical site infection (SSI) is a major contributor to obstetrical morbidity, hospital readmission, and higher health care cost. ${ }^{3}$ Interventions which prevent SSI could have a significant impact on maternal health as well as the cost of maternity care. ${ }^{4}$ Factors that are known to affect incisional SSI include surgery type, administration of perioperative antibiotic prophylaxis, and patient's comorbidities such as obesity. ${ }^{5}$ The relationship between postoperative wound care, especially wound dressing types, and SSI remains to be elucidated. ${ }^{6}$ Recently, a postoperative dressing that integrates the antimicrobial properties of chlorhexidine gluconate (CHG; $4 \%$ by weight), the practicality of absorbency, and the convenience of transparency has been approved by the Food and Drug Administration. The dressing has antimicrobial (CHG crosses microorganisms' outer membrane, and subsequently, attacks the bacterial cytoplasmic or inner membrane or the yeast plasma membrane) and absorption properties, and is transparent and adherent, making it useful in the obstetrical population. As there are no level 1 clinical trials focusing on these novel dressing types, and as the risk factors and pathogenesis for postcesarean SSI may not mirror other surgical procedures, such evidence specific to pregnancy is needed before extrapolating the results from other types of surgeries to cesarean procedures. At the same time, obesity, a known risk factor for SSI, has become an epidemic. ${ }^{7}$ In addition, it is important to consider health-related quality of life (HRQoL) and wellbeing in the context of health and disease treatment. Growing evidence ${ }^{8-10}$ supports that health is a multidimensional concept and health care treatments must incorporate domains related to physical, mental and emotional, and social functioning. HRQoL focuses beyond the direct measures of health and incorporates the quality-of-life consequences of health status as well as the concept of well-being, to assess a person's emotions and life satisfaction. We believe that both well-being and HRQoL have an important impact in maternal outcomes particularly during the postpartum period while recovering from a cesarean delivery and receiving wound care.

Hence, our aim was to evaluate the effect of a novel antimicrobial dressing on patient's satisfaction and HRQoL before beginning the pivotal trial about its efficacy in decreasing SSI rates.

\section{Materials and Methods}

\section{Study Design and Participants}

This was a single-center, randomized, controlled, open-label trial. Eloquest Healthcare, Inc., MI funded the trial but had no role in the study design, conduct, analysis, or reporting. Perinatal Research Division, The University of Texas Medical Branch and the principal investigator (A.F.S.) coordinated the study, data collection, management, and analysis independently from the sponsor. The institution's ethics committee approved the study. Before randomization, written informed consent was obtained from all participating subjects. All the authors back the accuracy and completeness of the data, and adherence of the study to the protocol. This study was reported according to CONSORT (Consolidated Standards of Reporting Trials) guidelines ${ }^{11}$ and registered at ClinicalTrials. gov (NCT03887299) before enrollment started.

All pregnant women who were at $24^{0 / 7}$ weeks or more of gestation with whom a decision to perform a cesarean was made and an admission body mass index $\geq 35 \mathrm{~kg} / \mathrm{m}^{2}$ were screened for eligibility. Subjects unwilling or unable to provide consent, with known allergy to chlorhexidine, with chorioamnionitis, active skin infection, and coagulopathy, incarcerated, immunosuppressed (taking systemic immunosuppressant or steroids [e.g., transplant subjects, not including steroids for lung maturity], HIV with CD $<200$, or other), whose plan of care was not to have skin closure (e.g., secondary wound closure, mesh closure), or there was high likelihood of additional surgical procedure beyond cesarean (e.g., scheduled hysterectomy, bowel, or adnexal surgery) were excluded. All patients received surgical prophylaxis within 60 minutes before a cesarean section according to our institution's guidelines.

\section{Randomization}

Pregnant women who met eligibility criteria and consented were randomly assigned in a 1:1 ratio to either standard dressing (STANDARD) or ReliaTect Post-Op Dressing with CHG (RELIATECT) before walking back to the operating room. Surgeons were aware of the assignment after wound incision closure. The randomization sequence was created independently using a computer-generated randomization and was concealed in opaque envelopes from those responsible for recruiting participants into the study. Blinding was not possible due to the nature of the intervention.

\section{Interventions}

Women assigned to the STANDARD group received wound dressing and postpartum care as per our current practice. Compression dressing consisting of gauze, Telfa, and adhesive tape was placed immediately after skin closure under sterile conditions. Dressing was removed after 24 hours from surgery completion and subjects had an absorption pad with overlying garments for the remaining postoperative days until standard postoperative visit for wound check (postoperative days 5-7).

Women assigned to RELIATECT group had ReliaTect PostOp Dressing with CHG applied immediately after skin closure as per the manufacturer's instructions under sterile conditions (-Fig. 1A). All surgeons underwent training on appropriate application of the ReliaTect Post-Op Dressing before initiation of the trial. Unless otherwise indicated (e.g., bleeding, reoperation), the dressing was to remain in place until the postoperative clinic visit on postoperative days 5 to 7 (-Fig. 1B, C).

The remainder of the subjects' intraoperative and postoperative care was similar for both arms and followed current standard clinical practice at our center, including extended-spectrum preoperative antibiotic prophylaxis. 


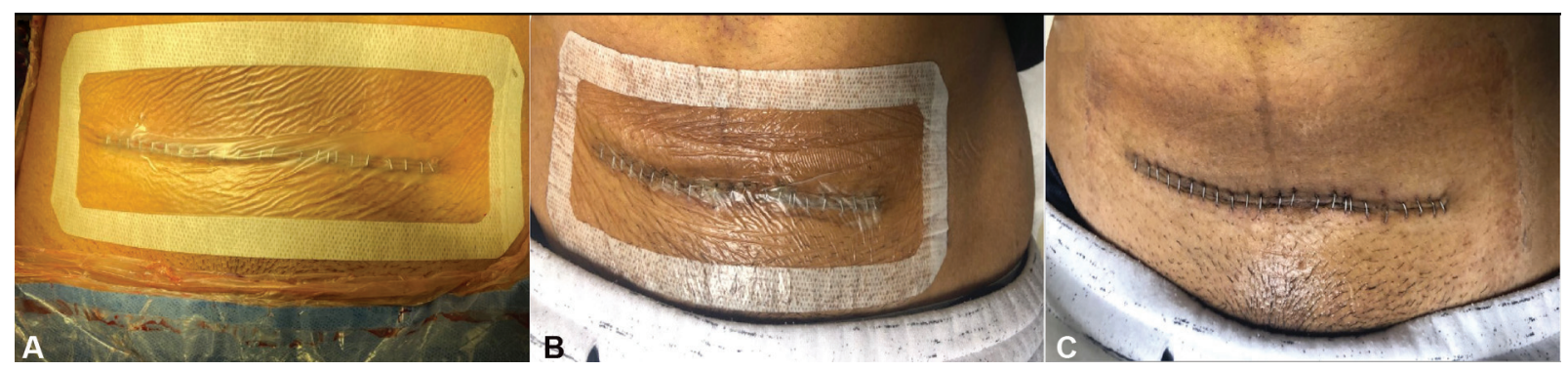

Fig. 1 (A-C) Illustration of the ReliaTect Post-Op Dressing at postoperative day 0 (A) and 7 before (B) after dressing removal (C).

Wound incision, skin, and subcutaneous fat tissue closure were all left at the surgeon's discretion. At our institution, skin closure is either performed with staples or with 3-0 poliglecaprone 25 sutures (Monocryl, Ethicon, Somerville, $\mathrm{NJ}$ ). Suture closure of subcutaneous fat was performed when the fat thickness was more than $2 \mathrm{~cm}$.

Trained and certified research staff members abstracted data from the medical records. It included demographics, medical history, and relevant outcomes. Participants were interviewed by research personnel immediately postpartum and completed a satisfaction survey at wound visit on postoperative days 5 to 7 .

\section{Primary and Secondary Outcomes}

The primary outcome was patient satisfaction and HRQoL questionnaire. ${ }^{12}$ Prespecified secondary outcomes were composite wound complication defined as presence of any of the following within 30 days from surgery by real time and/or chart review: SSI (defined per the Centers for Disease Control and Prevention [CDC] guidelines ${ }^{13}$ ), wound hematoma, seroma, or breakdown alone. Other secondary outcomes included provider satisfaction survey, endometritis, other infections (abscess, septic thrombosis, pneumonia, pyelonephritis, and breast infection), maternal death, puerperal fever (temperature $>100.4^{\circ} \mathrm{F}$ after first 24 hours or $\geq 101^{\circ} \mathrm{F}$ any time), postpartum antibiotic use, and allergic reactions (anaphylaxis, angioedema, skin rashes including StevensJohnson's syndrome and toxic epidermal necrolysis).

Patient satisfaction and HRQOL were assessed using a validated survey ${ }^{12}$ that was completed by subjects in both groups at their postoperative day wound clinic visit (postoperative days 5-7). This survey was modified and later validated to our target population. The questionnaire consisted of 11 questions that were scored on a 5-point Likert scale. ${ }^{14}$ The paper survey was in English or Spanish. Provider satisfaction was obtained from clinical providers who rounded in the patient while in house at the time of discharge on postoperative days 1 to 2 . Most providers and surgeons consisted of residents in obstetrics postgraduate years 1 to 4 .

\section{Sample Size Calculation}

We further validated the satisfaction and HRQoL questionnaire by surveying 20 obese women who underwent cesarean delivery before the study started and received the standard dressing. The mean score was 46 with a standard deviation of 9.1. Thus, a total sample size of 160 women was required to detect a difference of $10 \%$ in the primary outcome (power of $90 \%$ and two-sided $\alpha$ of $0.05,10 \%$ loss to follow-up).

\section{Statistical Analysis}

The primary analysis was conducted on an intention-to-treat basis. Between-group differences in continuous variables were assessed using Student's t-test or Mann-Whitney's rank-sum test, and results were given as mean, standard deviation or median, range as appropriate. Categorical variables were assessed using Pearson's chi-square test or Fisher's exact test. For dichotomous end points, relative risk (RR) and $95 \%$ confidence interval $(\mathrm{CI})$ values and for continuous end points, difference in the means and $95 \% \mathrm{CI}$ values were calculated. A $p$-value of $<0.05$ was considered significant.

The analysis was performed after data lock by an independent research staff, blinded to the group assignment. Blinded analysis was performed for the primary outcome and then unblinded after analysis was completed.

Statistical analyses were performed using STATA 16 software (StataCorp, College Station, TX) and GraphPad Prism version 8.0.0 for Mac (GraphPad Software, San Diego, CA).

\section{Results}

\section{Participants and Study Design}

From April 2019 to January 2020, a total of 1,177 women were screened for eligibility; 155 women consented and were randomized (78 in RELIATECT; 77 in STANDARD) ( - Fig. 2). One participant in the RELIATECT group was consented but was found to be ineligible after randomization, secondary to intraoperative finding of an infection at the planned skin incision site; hence, this subject was not included in the analysis and did not receive the assigned intervention. ${ }^{15}$ Protocol deviations occurred in nine participants for a total protocol adherence of $94 \%$ (better than the estimated protocol adherence of $90 \%$ ). Majority of protocol deviations consisted of wound dressing being removed earlier than planned. Baseline characteristics at randomization were similar among groups (-Table 1). Our patient population consisted mainly of multiparous, young obese white Hispanic pregnant women. There were no differences in the rates of postpartum hemorrhage, vertical skin incision, and skin closure with staples.

\section{Primary and Secondary Outcomes}

- Table 2 summarizes survey scores, and -Fig. 3 illustrates statistical significant distribution histograms of the primary 


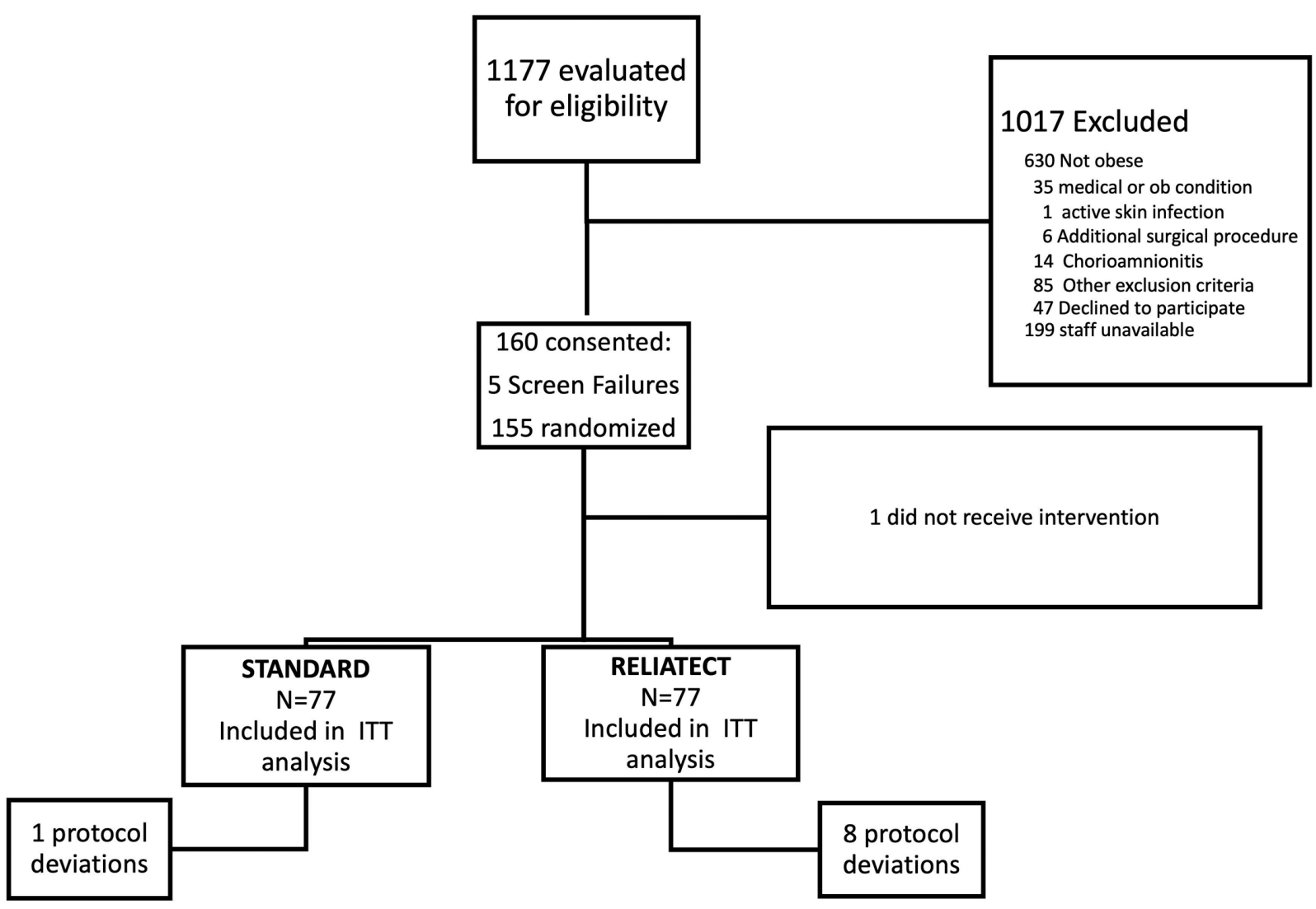

Fig. 2 Flow diagram.

outcome stratified by scores of 5 or more ("very happy") versus 4 or less ("less happy"). Obese women in the RELIATECT group had an overall higher satisfaction and HRQoL score compared with the STANDARD group (52 vs. 49; mean difference [MD]: 3.27; 95\% CI [0.78-5.76]; $p=0.002)$. Women in the RELIATECT group reported less incision odor (4 [15] vs. 5 [1-5]; MD: $0.15 ; 95 \%$ CI [0.08-0.69]; $p=0.0002)$ ( - Table 2 and -Fig. 3), and incisional pain (4 [1-5] vs. 5 [15]; MD: 0.44; 95\% CI [0.10-0.77]; $p=0.009)$. Compared with the STANDARD group, most women in the RELIATECT group reported better daily activities (5 [1-5]) vs. 5 [1-5]; MD: $0.42 ; 95 \% \mathrm{CI}[0.10-0.75] ; p=0.007$ ), self-esteem (5 [1-5] vs. 5 [1-5]; MD: 0.34; 95\% CI [0.06-0.63]; $p=0.018)$, personal hygiene (5 [1-5] vs. 5 [1-5]; MD: 0.32; 95\% CI [0.04-0.60]; $p=0.003$ ), body image (5 [1-5] vs. 5 [1-5]; MD: $0.40 ; 95 \% \mathrm{Cl}$ [0.08-0.72]; $p=0.007$ ), and sleep (4 [1-5] vs. 5 [1-5]; MD: 0.53; 95\% CI [0.19-0.88]; $p=0.001$ ) (-Table 2 and -Fig. 3 ).

Providers reported that the RELIATECT dressing allowed better assessment of the surgical incision site (2 [1-5] vs. 5 [1-5]; MD: 1.78; 95\% CI [1.33-2.23]; $p<0.0001)$, allowed patients to shower early (2 [1-5] vs. 4 [1-5]; MD: $1.42 ; 95 \% \mathrm{CI}$ [0.98-1.85]; $p<0.0001)$, and did observe less wound dressing leakage (4 [1-5] vs. 5 [1-5]; MD: 0.54; 95\% CI [0.12-0.98]; $p=0.003)$ (-Table 3 ).

Other secondary outcomes were not different between the groups ( - Table 4 ), SSI rates did not differ among groups (STANDARD: 1 [1.29\%] vs. RELIATECT: 2 [2.59\%]; RR: 2; 95\% CI [0.18-21.60]; $p=0.568$ ). The rates of outpatient antibiotic administration within 30 days of surgery were similar among both dressing methods (STANDARD: 5 [6.49\%] vs. RELIATECT groups: 5 [6.49\%]; RR: 1 ; 95\% CI [0.30-3.32]; $p=1$ ).

\section{Discussion}

\section{Principal Findings}

We found that obese women receiving postpartum wound care with RELIATECT dressing after cesarean delivery were more satisfied and reported a higher HRQoL activity scores compared with those who received standard wound care. Providers' feedback toward the novel dressing was also positive. Although not powered for secondary outcomes, the rates of SSI and wound complications did not differ among groups. Given our findings, RELIATECT novel dressing is not inferior to standard of care dressing with better patient and provider satisfaction.

\section{Results}

Growing proof ${ }^{8-10}$ supports that health is a multidimensional concept and health care treatments must incorporate domains related to physical, mental and emotional, and social functioning. HRQoL focuses beyond the direct measures of health and incorporates the quality-of-life consequences of health status as well as the concept of well-being, to assess a person's emotions and life satisfaction. During wound care, mothers feel distressed and are worried about pain control, wound incision healing, and ultimately, the ability to return to daily activities, hence the importance of well-being and HRQoL when evaluating wound care after a 


\begin{tabular}{|c|c|c|}
\hline $\begin{array}{l}\text { Population } \\
\text { characteristics (ITT) }\end{array}$ & $\begin{array}{l}\text { STANDARD } \\
(n=77)\end{array}$ & $\begin{array}{l}\text { RELIATECT } \\
(n=77)\end{array}$ \\
\hline Age (y) & $29(20-41)$ & $29(19-41)$ \\
\hline Gestational age (wk) & $39(32-39)$ & $39(34-40)$ \\
\hline Gravidity & $3(1-9)$ & $3(1-7])$ \\
\hline Parity & $1(0-7)$ & $2(0-5])$ \\
\hline Maternal weight $(\mathrm{kg})$ & $111(82-208)$ & $102(82-182)$ \\
\hline BMI & $40(31-79)$ & $40(34-62)$ \\
\hline BMI $\left(>35 \mathrm{~kg} / \mathrm{m}^{2}\right)$ & $76(98.7)$ & $76(98.7)$ \\
\hline BMI $\left(>40 \mathrm{~kg} / \mathrm{m}^{2}\right)$ & $41(53.2)$ & $46(59.7)$ \\
\hline No. of prior cesareans & $1(0-6)$ & $1(0-4)$ \\
\hline \multicolumn{3}{|l|}{ Race } \\
\hline White & $62(80.5)$ & $61(79.2)$ \\
\hline African American & $14(18.1)$ & $16(20.7)$ \\
\hline $\begin{array}{l}\text { Native American/ } \\
\text { Hawaiian }\end{array}$ & $1(0.01)$ & $0(0)$ \\
\hline Hispanic & $37(48.0)$ & $47(61.0)$ \\
\hline Gestational diabetes & $13(16.8)$ & $5(6.49)$ \\
\hline A1 GDM & $3(3.89)$ & $2(2.59)$ \\
\hline A2 GDM (oral) & $7(9.09)$ & $2(2.59)$ \\
\hline A2 GDM (insulin) & $3(3.89)$ & $0(0)$ \\
\hline \multicolumn{3}{|l|}{ Medical conditions } \\
\hline None & $50(64.9)$ & $54(70.1)$ \\
\hline Pregestational diabetes & $7(9.09)$ & $4(5.19)$ \\
\hline Preeclampsia & $3(3.89)$ & $1(1.29)$ \\
\hline $\begin{array}{l}\text { Preeclampsia with } \\
\text { severe features }\end{array}$ & $2(2.59)$ & $3(3.89)$ \\
\hline Chronic hypertension & $12(15.5 \%)$ & $11(14.3 \%)$ \\
\hline Combination of above & $3(3.89)$ & $4(5.19)$ \\
\hline $\begin{array}{l}\text { Other complications } \\
\text { of pregnancy }\end{array}$ & $1(1.29)$ & $3(3.89)$ \\
\hline \multicolumn{3}{|l|}{ Delivery complications } \\
\hline None & $64(83.11)$ & $69(89.6)$ \\
\hline $\begin{array}{l}\text { Postpartum } \\
\text { hemorrhage }\end{array}$ & $11(14.2)$ & $6(7.79)$ \\
\hline Other & $1(1.29)$ & $2(2.59)$ \\
\hline Vertical skin incision & $8(10.3)$ & $6(7.79)$ \\
\hline Skin closure with staples & $44(57.1)$ & $43(55.8)$ \\
\hline
\end{tabular}

Abbreviations: BMI, body mass index; GDM, gestational diabetes mellitus; ITT, intent to treat.

Note: Data are presented as $n$ (\%) or median (range).

a Demographics and baseline characteristics were analyzed in the ITT population, and no significant differences were noted among groups.

cesarean delivery. This is the first published trial evaluating these measurements among two types of wound care dressings. Patients and providers were more satisfied with the RELIATECT compared with the STANDARD. Moreover, women in the RELIATECT had better HRQoL scores; such as, better daily activities, self-esteem, personal hygiene, body image, and better sleep. This is likely due to the difference in surgical wound care; rather to have the skin exposed, the RELIATECT covers the surgical site for a full 5 to 7 days, it is transparent and allows ease of movement and ability to perform daily activities without patients worrying of changing dressing or of the wound touching surrounding garments or leaking fluid. The dressing did not impact negatively daily activities and patients reported better self-esteem, better personal hygiene, better body image, and better sleep.

Neither dressing had major adverse events, such as wound dehiscence, deep or superficial SSI per CDC guidelines, ${ }^{13}$ wound seroma, wound hematoma, endometritis nor other comorbidities. Despite higher rates of antimicrobial use in the RELIATECT group, this was not statistically different, and it was initiated for nonclinical indications such as visualization of exudate film through transparent dressing, which is an expected finding with the RELIATECT dressing. This finding may be secondary to providers' unfamiliarity with the new dressing and may have been avoided with better education of providers to expectations of the RELIATECT dressing. Still, the safety profile for RELIATECT was not significantly different from STANDARD.

Wound complications in the obstetrical population ranges between 2 and 30\%. ${ }^{16}$ SSI after a cesarean section has been associated with adverse maternal outcomes, prolonged hospital stay, and increased health care costs. ${ }^{4}$ Early detection of relevant risk factors such as obesity, diabetes, smoking, peripartum infections, immunosuppression, and prolonged rupture of membranes are crucial for SSI prevention and management. One of the $\mathrm{CDC}^{16,17}$ recommendations for SSI prevention is covering the incision site with a sterile dressing for 24 to 48 hours. This was based on an animal study ${ }^{18}$ that showed wound resurfacing was completed $>24$ hours. The optimal wound care or postoperative dressing that decreases SSI rates leads to better patient satisfaction, better HRQoL, and less scarring remains undetermined. For example, Cochrane reviews ${ }^{6,19}$ did not show differences in scar, SSI, pain, or patient's acceptability among different postoperative dressings. Furthermore, some critics even argue against the use of any postoperative dressing due to the lack of evidence. ${ }^{6,20}$ The ReliaTect PostOp Dressing with CHG was designed to address these concerns. We designed this clinical trial (the first to evaluate this dressing) to primarily assess patient's acceptability and in addition to use its preliminary data to power a larger trial to assess its effect on wound complications and SSI rates.

\section{Strengths and Limitations}

The strengths of our study include its randomized design, prespecified outcomes and analyses, and management of wound care that is consistent within a single institution. In addition, the patients were managed independently from the investigators. Our study has also some limitations. Given the nature of the intervention, masking was not an option. This may have led to higher scores in patients who received the novel dressing. We tried to limit further bias by locking the 


\begin{tabular}{|c|c|c|c|}
\hline $\begin{array}{l}\text { Primary outcome (ITT) } \\
\text { Patient satisfaction }\end{array}$ & STANDARD $(n=77)$ & RELIATECT $(n=77)$ & Difference $(95 \% \mathrm{Cl})$ \\
\hline $\begin{array}{l}\text { Odor (bad smell) of the incision wound from } \\
\text { your cesarean delivery }\end{array}$ & $4(1-5)$ & $5(1-5)$ & $0.15(0.08-0.69)$ \\
\hline $\begin{array}{l}\text { Leakage of the incision wound from your } \\
\text { cesarean delivery }\end{array}$ & $5(1-5)$ & $5(1-5)$ & $0.07(-0.21$ to 0.36$)$ \\
\hline $\begin{array}{l}\text { Itching of the incision wound from your } \\
\text { cesarean delivery }\end{array}$ & $5(1-5)$ & $5(1-5)$ & $-0.03(-0.36$ to 0.29$)$ \\
\hline $\begin{array}{l}\text { The way my wound dressing affected my daily } \\
\text { activities (ability to wear pants or garment } \\
\text { that goes across the incision) or movements }\end{array}$ & $5(1-5)$ & $5(1-5)$ & $0.42(0.10-0.75)$ \\
\hline $\begin{array}{l}\text { The way my wound dressing affected my } \\
\text { body image }\end{array}$ & $5(1-5)$ & $5(1-5)$ & $0.40(0.08-0.72)$ \\
\hline $\begin{array}{l}\text { The way my wound dressing affected } \\
\text { my self-esteem }\end{array}$ & $5(1-5)$ & $5(1-5)$ & $0.34(0.06-0.63)$ \\
\hline $\begin{array}{l}\text { The way my wound dressing affected my } \\
\text { personal hygiene }\end{array}$ & $5(2-5)$ & $5(1-5)$ & $0.32(0.04-0.60)$ \\
\hline $\begin{array}{l}\text { The ability of the wound dressing to } \\
\text { protect my wound (incision site) }\end{array}$ & $5(1-5)$ & $5(1-5)$ & $0.13(-0.18$ to 0.44$)$ \\
\hline The way my wound dressing affected my sleep & $4(1-5)$ & $5(1-5)$ & $0.53(0.19-0.88)$ \\
\hline $\begin{array}{l}\text { The way my wound dressing affected my } \\
\text { incisional pain }\end{array}$ & $4(1-5)$ & $5(1-5)$ & $0.44(0.10-0.77)$ \\
\hline $\begin{array}{l}\text { Overall I am satisfied with my postoperative } \\
\text { wound dressing care }\end{array}$ & $5(2-5)$ & $5(1-5)$ & $0.18(-0.08$ to 0.43$)$ \\
\hline Total score & $49(20-55)$ & $52(12-55)$ & $3.27(0.78-5.76)$ \\
\hline
\end{tabular}

Abbreviations: $\mathrm{Cl}$, confidence interval; ITT, intent to treat; HRQoL, health-related quality of life. Note: Data are presented as median (range).
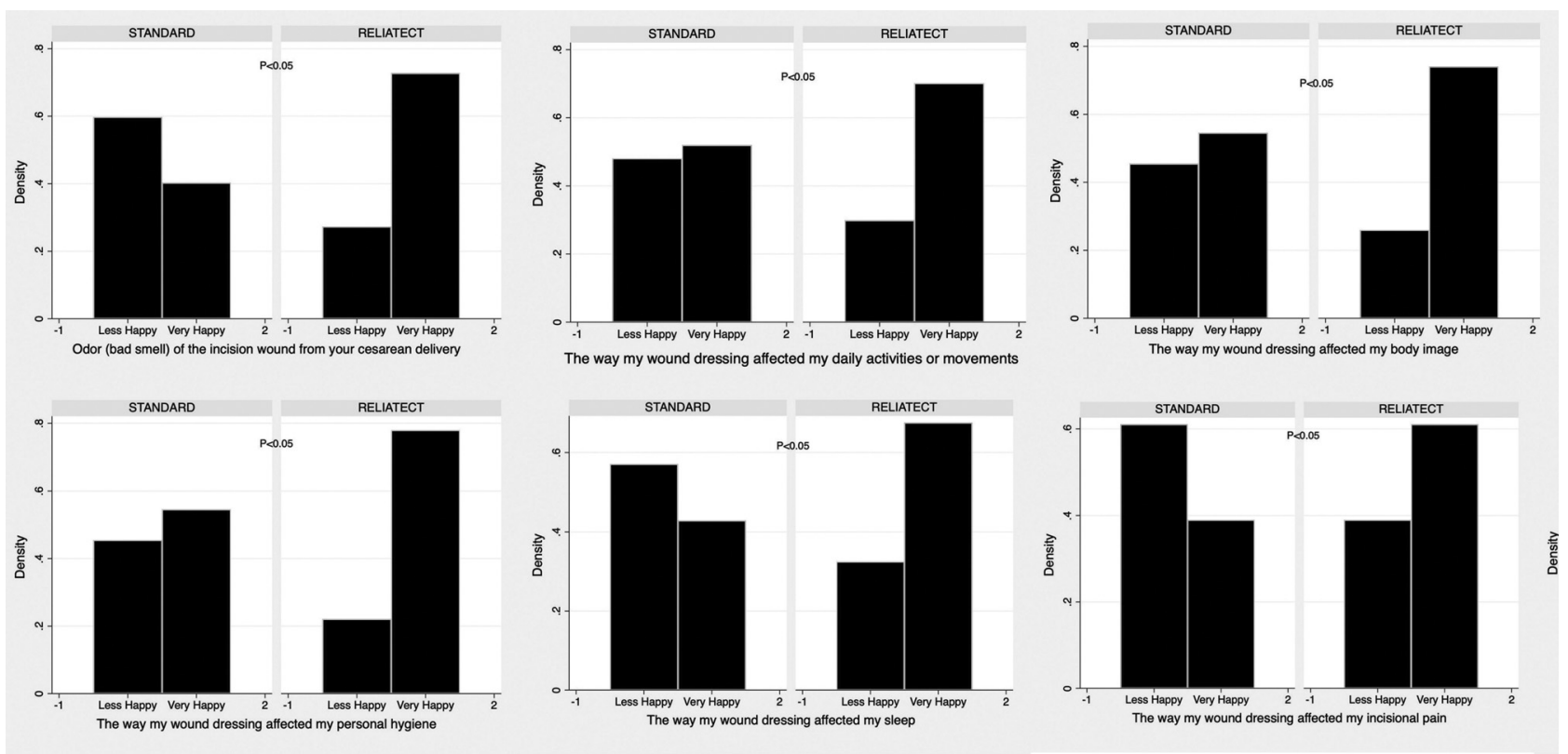

Fig. 3 Survey: distribution histograms. Answers categorized by scores of 5 or more ("very happy") versus scores less than 5 ("less happy"). Y-axis reflects proportions.

dataset prior to the analysis, which was performed by personnel who were blinded to the group assignment. Another limitation is that a study in a single center may have lower variability in management and therefore may be less generalizable to other centers. However, determining whether the results of a trial are applicable to other patient population is not unique to ours. Since our trial was not powered to assess SSI outcomes, our study cannot elucidate 


\begin{tabular}{|c|c|c|c|}
\hline $\begin{array}{l}\text { Secondary outcome (ITT) } \\
\text { Provider satisfaction }\end{array}$ & STANDARD $(n=74)$ & RELIATECT $(n=75)$ & Difference $(95 \% \mathrm{Cl})$ \\
\hline Dressing allowed wound to be assessed & $2(1-5)$ & $5(1-5)$ & $1.78(1.33-2.23)$ \\
\hline Dressing did not allow leakage & $4(1-5)$ & $5(1-5)$ & $0.54(0.12-0.98)$ \\
\hline Dressing allowed patient to shower early & $2(1-5)$ & $4(1-5)$ & $1.42(0.98-1.85)$ \\
\hline $\begin{array}{l}\text { Dressing stayed in place/secure for the time } \\
\text { required/desired }\end{array}$ & $5(1-5)$ & $5(1-5)$ & $0.22(-0.09$ to 0.53$)$ \\
\hline $\begin{array}{l}\text { No signs/symptoms of infection at time } \\
\text { of assessment }\end{array}$ & $5(1-5)$ & $5(1-5)$ & $0.28(0.01-0.54)$ \\
\hline Total score & $17(5-25)$ & $21(5-25)$ & $4.14(2.65-5.63)$ \\
\hline
\end{tabular}

Abbreviations: $\mathrm{Cl}$, confidence interval; ITT, intent to treat.

Note: Data are presented as median (range).

\begin{tabular}{|c|c|c|c|}
\hline Secondary outcomes (ITT) & STANDARD $(n=77)$ & RELIATECT $(n=77)$ & Relative risk $(95 \% \mathrm{Cl})$ \\
\hline Surgical site infection ${ }^{a}$ & $1(1.29)$ & $2(2.59)$ & $2(0.18-21.60)$ \\
\hline Postpartum endometritis & $0(0)$ & $1(1.29)$ & \\
\hline Other wound complication & $5(6.5)$ & $9(13.2)$ & $2(0.71-5.58)$ \\
\hline Antimicrobial given ${ }^{\mathrm{b}}$ & $4(5.19)$ & $6(7.79)$ & \\
\hline Allergic reaction & $0(0)$ & $1(1.29)$ & \\
\hline Wound dehiscence & $1(1.29)$ & $0(0)$ & \\
\hline Other ${ }^{c}$ & $0(0)$ & $2(2.59)$ & \\
\hline Readmission $^{d}$ & $0(0)$ & $3(3.89)$ & \\
\hline Postpartum triage visit & $10(12.9)$ & $6(7.79)$ & $0.6(0.22-1.56)$ \\
\hline Outpatient antibiotics $^{\mathrm{e}}$ & $5(6.49)$ & $5(6.49)$ & $1(0.30-3.32)$ \\
\hline Postpartum antibiotics before Discharge & $1(1.29)$ & $1(1.29)$ & $1(0.06-15.70)$ \\
\hline
\end{tabular}

Abbreviations: $\mathrm{Cl}$, confidence interval; ITT, intent to treat.

Note: Data are presented as $n(\%)$.

${ }^{a}$ Defined per CDC guidelines.

${ }^{b}$ For suspected wound infection at any time after delivery.

'Two readmissions were due to wound infection, one was for preeclampsia.

${ }^{\mathrm{d}}$ Combination of one or more of the above wound complications.

'Within 30 days of delivery for any indication.

whether this novel dressing is superior to current practice in decreasing wound infection rates.

\section{Clinical Implications}

ReliaTect Post-Op Dressing with CHG wound care in obese women undergoing cesarean delivery led to better patient and provider satisfaction as well as better HRQoL. While both groups had minimal wound complications and SSI events, advantages of RELIATECT dressing include antimicrobial properties, practicality of absorbency, and the convenience of transparency. Further, level 1 evidence would allow us to assess its impact on SSI rates and wound complication, as this trial was not powered to accomplish this goal. We hope that our results will pave the way to further clinical application studies elucidating ReliaTect's role in postcesarean wound care, most importantly, its efficacy in decreasing SSI and wound complication rates to justify its cost compared with standard dressing.

\section{Conclusion}

Postcesarean RELIATECT dressing for wound care in pregnant women with obesity had better patient and provider satisfaction as well as better HRQoL scores, such as better daily activities, self-esteem, personal hygiene, body image, and sleep.

\section{Clinical Trial Registration}

ClinicalTrials.gov; date of registration: March 22, 2019; date of first enrollment: April 18, 2019; available at: https://clinicaltrials.gov/ct2/show/NCT03887299

\section{Authors' Contributions}

A.F.S., A.E.S., and G.R.S. conceived the study and editing the final manuscript. A.F.S. and G.R.S. were involved in data analysis, interpretation of data, drafting of manuscript, and critical review and editing the manuscript. A.F.S. and L.A. were involved in data abstraction/extraction. 
L.A. was involved in data abstraction. All authors approved the final version for submission.

\section{Note}

After review and determination by the University of Texas Medical Branch Galveston Hospital Institutional Review Board (Name: "Institutional Review Board," 301 University Boulevard, Galveston, TX 77550-0158), our research project (IRB protocol \# 18-0265) was found to meet the criteria for approval on March 8, 2019. Written informed consent was obtained from all participants prior to inclusion and randomization in the study. Participants were identified by number, not by name.

\section{Funding}

This trial was funded by Eloquest Healthcare, Inc., MI. The funder did not have any role in the conduct of the trial, analysis of the data, or drafting of this manuscript.

\section{Conflict of Interest}

None declared.

\section{Acknowledgments}

The authors would like to thank the labor and delivery staff, MFM faculty, and residents at our facility.

\section{References}

1 Betrán AP, Ye J, Moller AB, Zhang J, Gülmezoglu AM, Torloni MR. The increasing trend in caesarean section rates: global, regional and national estimates: 1990-2014. PLoS One 2016;11(02):e0148343

2 Benson J. Preventing the first cesarean delivery: summary of a joint Eunice Kennedy Shriver National Institute of Child Health and Human Development, Society for Maternal-Fetal Medicine, and American College of Obstetricians and Gynecologists Workshop. Obstet Gynecol 2013;121(04):878-879

3 Kirkland KB, Briggs JP, Trivette SL, Wilkinson WE, Sexton DJ. The impact of surgical-site infections in the 1990s: attributable mortality, excess length of hospitalization, and extra costs. Infect Control Hosp Epidemiol 1999;20(11):725-730

4 NICE Guideline Updates Team. Surgical site infections: prevention and treatment. London, UK: National Institute for Health and Care Excellence; 2019

5 Gibbs RS. Clinical risk factors for puerperal infection. Obstet Gynecol 1980;55(05):178S-184S
6 Walter CJ, Dumville JC, Sharp CA, Page T. Systematic review and meta-analysis of wound dressings in the prevention of surgicalsite infections in surgical wounds healing by primary intention. $\mathrm{Br}$ J Surg 2012;99(09):1185-1194

7 Poston L, Caleyachetty R, Cnattingius S, et al. Preconceptional and maternal obesity: epidemiology and health consequences. Lancet Diabetes Endocrinol 2016;4(12):1025-1036

8 Schwartz CE, Andresen EM, Nosek MA, Krahn GLRRTC Expert Panel on Health Status Measurement. Response shift theory: important implications for measuring quality of life in people with disability. Arch Phys Med Rehabil 2007;88(04):529-536

9 Ostir GV, Markides KS, Black SA, Goodwin JS. Emotional wellbeing predicts subsequent functional independence and survival. J Am Geriatr Soc 2000;48(05):473-478

10 Pressman SD, Cohen S. Does positive affect influence health? Psychol Bull 2005;131(06):925-971

11 Schulz KF, Altman DG, Moher DCONSORT Group. CONSORT 2010 statement: updated guidelines for reporting parallel group randomized trials. Obstet Gynecol 2010;115(05):1063-1070

12 Manoharan V, Grant AL, Harris AC, Hazratwala K, Wilkinson MP, McEwen PJ. Closed incision negative pressure wound therapy vs conventional dry dressings after primary knee arthroplasty: a randomized controlled study. J Arthroplasty 2016;31(11): 2487-2494

13 Horan TC, Gaynes RP, Martone WJ, Jarvis WR, Emori TG. CDC definitions of nosocomial surgical site infections, 1992: a modification of CDC definitions of surgical wound infections. Am J Infect Control 1992;20(05):271-274

14 Davey HM, Barratt AL, Butow PN, Deeks JJ. A one-item question with a Likert or visual analog scale adequately measured current anxiety. J Clin Epidemiol 2007;60(04):356-360

15 Fergusson D, Aaron SD, Guyatt G, Hébert P. Post-randomisation exclusions: the intention to treat principle and excluding patients from analysis. BMJ 2002;325(7365):652-654

16 Mangram AJ, Horan TC, Pearson ML, Silver LC, Jarvis WRHospital Infection Control Practices Advisory Committee. Guideline for prevention of surgical site infection, 1999. Infect Control Hosp Epidemiol 1999;20(04):250-278, quiz 279-280

17 Black JD, de Haydu C, Fan L, Sheth SS. Surgical site infections in gynecology. Obstet Gynecol Surv 2014;69(08):501-510

18 Eaglstein WH, Davis SC, Mehle AL, Mertz PM. Optimal use of an occlusive dressing to enhance healing. Effect of delayed application and early removal on wound healing. Arch Dermatol 1988; 124(03):392-395

19 Dumville JC, Gray TA, Walter CJ, et al. Dressings for the prevention of surgical site infection. Cochrane Database Syst Rev 2016;12 (12):CD003091

20 Borkar NB, Khubalkar MV. Are postoperative dressings necessary? J Wound Care 2011;20(06):301 